\title{
Dangers of Using Skin Whitening Products in the Cosmetic Market of Bangladesh
}

\author{
Rahman $\mathrm{MH}^{1}$
}

Skin whitening or lightening or bleaching is a cosmetic procedure that aims to lighten dark areas of skin or achieve a generally paler or brighter skin complexion. While a whiter skin complexion is still considered desirable in our culture, more and more woman are seeking skin whitening treatment. Many powerful skin-lightening creams are available in the market of Bangladesh. But despite the risks, dangerous ingredients are used in beauty treatments in our country - like mercury, steroid and hidroquinone are still be found in skin whitening creams and soaps today. Products containing these ingredients that are banned in the UK, as they can cause serious side effects if used incorrectly. ${ }^{1}$

Skin whitening or skin lightening creams can be used to treat a range of problems including dark spots, acne scarring, freckles and melasma but care should be taken when choosing such a product. Many skin lighteners still contain chemicals that can cause serious health risks if used for prolonged periods. Uneven skin tones and the areas of hyperpigmentation can occur as a result of sun damage and skin irritation., ${ }^{1,2}$

Melanin is a brown pigment produced in the melanocytes in the skin. If is the body's natural protection against the harmful effects of UV rays. However, an over production of melanin (hyperpigmentation) can cause uneven skin tones and other skin disorders. ${ }^{3}$

Skin bleaching agents act to reduce the action of the enzyme, tyrosinase, which controls the rate of melanin production in the skin. Skin whitening creams contain ingredients that act to slow the production of melanin in the skin's outer layer. ${ }^{4}$ Products that contain effective ingredients can help to treat the problems associated with hyperpigmentation, however, chemical-based skin whiteners can be potentially dangerous and leads to significant health risks with extended use. ${ }^{5}$ Chemicals traditionally used in skin whitening products can include hydroquinine, mercury and steroids. While the use of these chemicals has been banned in some countries, ingredients like hydroquinine may still be prescribed by physicians. ${ }^{5}$

The biggest danger of exposing the skin and body to these toxic chemicals is that, over time, they can produce adverse side effects and serious health concerns including skin discoloration, malfunctions of the nervous system and internal organs and an increased risk of cancer. ${ }^{6}$ Common side effects reported from the use of hydroquinine-creams include skin rashes, burning skin irritation, excessive redness and a dryness or cracking of the skin.

Since we do not know the precise extent of the health risks hydroquinone poses, it may be considered to be cytotoxic (toxic to cells), mutagenic and carcinogenic (cancer causing). Hydroquinine is thought to increase the risk of complications such as thyroid disorders, liver disease and adrenal dysfunction.

If used for extended periods of time, hydroquinone can sometimes induce a condition known as "ochronosis." People with ochronosis show a blueblack darkening in certain areas of the skin. ${ }^{7}$ Like other pigment-reducing complexes, hydroquinone can also make skin more susceptible to the sun's UV rays. This can lead to serious sunburn and an increased risk of certain types of skin cancer.

\author{
1. *Prof. Dr. Md. Hasibur Rahman \\ Professor \& Head \\ Department of Skin \& VD \\ Community Based Medical College, Bangladesh. \\ * Address of correspondence \\ E-mail: dr_cosmoderma@yahoo.com \\ Mobile: +8801711318709
}


Mercury is another commonly used skin products which blocks the formation of melanin. It is also present in makeup as preservatives. It is fairly known that mercury is toxic and if present in water, it can contaminate fish. Ingestion of mercury poisoned fish by pregnant women leads to birth defects. Mercury products applied to the skin can cause kidney damage. $^{8}$

Mercury body a toxic chemical is readily absorbed into the body but it is not easily removed. When mercury is used for skin whitening the initial side effects can include skin rashes, skin discoloration and scarring. Long term exposure to mercury can damage the kidneys and the nervous system. It can also cause depression or psychosis and interfere with the development of the brain in unborn children and very young children. ${ }^{8}$

In August 2013 the Food and Drug Administration (FDA) issued a warning to consumers advising them not to use skin creams, beauty soaps or lotions that might contain mercury due to the dangers of mercury poisoning. The FDA first banned the use of mercury in skin-bleaching and lightening products as far back as in 1990 but the regulatory office has since found that mercury was being used as an ingredient in some products that were manufactured abroad and sold illegally in the United States.

The EU and many African countries have banned the usage of mercury in skin creams, however, there are no strict laws about this in Bangladesh.

Unfortunately, many skin-lightening creams contain illegal compounds which can include high-dose of steroids. Although steroids can be useful in treating inflammation of the skin caused by diseases such as eczema, psoriasis or dermatitis, but they were never intended for skin lightening use.

Steroids are to be prescribed under the supervision of a skin specialist and it must be rational.

While topical corticosteroids can appear to lighten the skin very quickly, this is because they act as "vaso-constrictors". This means that the blood vessels in the area treated will become constricted (narrow) and the flow of blood will be slowed, giving the skin a whiter appearance. ${ }^{9}$

Steroids can also slow the process of cell regeneration as a result less melanocytes are formed, leading to a decline in melanin production. The negative side of slowing the skin's natural cell renewal is that the epidermis (outer skin layer) can become thinner and many people complain of thinning of skin and the appearance of telangiectasia.

The use of high-dose steroids can lead to many more problems. The thinning effect on the skin can increase the risk of physical damage to the skin. The skin can become more susceptible to chemical and environmental factors and there will be an increased risk of sun damage and additional pigmentation problems.

The high doses of steroids found in the illegal skin whitening creams can also interfere with the body's hormone levels and, in extreme cases, can result in disorders such as "Cushings syndrome' which affects the adrenal gland. ${ }^{9}$

The obsession with fairness and skin lightening products in Bangladesh and, in fact, throughout most parts of Asia and Africa is rather freightening. ${ }^{10}$

In contrast to the above mentioned ingradients natural skin lightening products include ingredients such as arbutin, emblica, liquorice, mulberry extract, kojic acid or Vitamin $C$ can be used safely for skin lightening issues. ${ }^{11,12}$

So now the time has come to take necessary steps regarding the indiscriminate and injudicious use of fairness cream in our country.

\section{References:}

1. Katsambas, A.D.; Stratigos, A.J. Depigmenting and bleaching agents: Coping with hyperpigmentation. Clin. Dermatol. 2001, 19, 483-488.

2. Rendon, M.; Berneburg, M.; Arellano, I.; Picardo, M. Treatment of melasma. J. Am. Acad. Dermatol. 2006, 54, S272-S281. 
3. Barsh, Gregory S. (2003). "What Controls Variation in Human Skin Color?". PLoS Biology. 1: E27.

4. Ito, Shosuke; Wakamatsu, Kazumasa "A convenient screening method to differentiate phenolic skin whitening tyrosinase inhibitors from leukoderma-inducing phenols" J. Dermatol. Sci. (2015). 80 (1): 18-20

5. Papaspyrides, C.D.; Protopapas, S.A. E.s.r. approach on hydroquinone-melanin possible interaction. Int. J. Biol. Macromol. 1988, 10, 62-63.

6. O'Donaghue, J.L., David, P.; Richardson, W.; Dyer, M. Hydroquinone and hepatitis. Lancet 1995, 346, 1427-1428.

7. Tidman, M.J., Horton, J.J.; MacDonald, D.M. Hydroquinone-induced ochronosis-Light electronmicroscopic features. Clin. Exp. Dermatol. 1986, 11, 224-228.

8. Yetunde M. Olumide, Ayesha 0. Akinkugbe, Dan Altraide, Tahir Mohammed, Ngozi Ahamefule, Shola
Ayanlowo, Chinwe Onyekonwu, and Nyomudim Essen. Complications of chronic use of skin lightening cosmetics. International Journal of Dermatology 2008;7:344-353.

9. Corticosteroids (Ch. 25). In: Bolognia JL, Jorizzo JL, Rapini RP, editors. Bolognia Textbook of Dermatology. 2nd ed. Mosby Elsevier publishing; 2008.

10. McDougall, Andrew (June 4, 2013). "Skin lightening trend in Asia boosts global market". Cosmetics Design Asia.

11. Degen, G.H. Opinion of the Scientific Committee on Consumer Safety (SCCS)-Opinion on the safety of the use of -arbutin in cosmetic products. Regul. Toxicol. Pharmacol. 2016, 74, 75-76.

12. Zhu, W.; Gao, J. The Use of Botanical Extracts as Topical Skin-Lightening Agents for the Improvement of Skin Pigmentation Disorders. J. Investig. Dermatol. Symp. Proc. 2008, 13, 20-24. 